To the Editors:

\title{
Selection of students for admission to a medical school in Sri Lanka [1]
}

The area of research is timely since selection of students for admission to medical schools has received much attention recently. Selection criteria need to be examined in relation to outcome, success in the medical course being one such outcome. The population for such an investigation has necessarily to be the total cohort (or better still, cohorts) of students who gain entry to all medical schools in a given year (or years). This is so because of the differential distribution of the different "quotas" and criteria for selection in medical schools.

It is noted that in the medical school selected for study, the variation observed in the independent variables (predictors) is very small. This is likely to be a function of the distribution of the "quotas" in the selected school, highlighting the need for studying those who are admitted to all six medical schools as one group. Absence of information on some important predictors such as proficiency in English language and the number of attempts at the Advanced Level examination adds to the difficulties of examining this relationship.

The above aspects are mentioned in passing in the discussion. However, the limitations in generalizing the results to all admissions to medical schools are not sufficiently dealt with and thus, the article is liable to misinterpretation. This would harm a meaningful debate on the appropriateness of current admission criteria, negating the intentions of the researchers.

\section{Reference}

1. De Silva NR, Pathmeswaran A, de Silva HJ. Selection of students for admission to a medical school in Sri Lanka. Ceylon Medical Journal 2004; 49: 81-5.

Lalini Rajapaksa, Professor in Community Medicine, Faculty of Medicine, Kynsey Road, Colombo 8. E-mail: <lcraj@sltnet.lk> (Competing interests: none declared). 